\title{
Low FDG Metabolic Activity of Loco-Regional Recurrence in Thyroid Cancer Patients; Is Iodine-131 has a Potentiality?
}

\author{
Amr Amin ${ }^{*}, 1,3$, Mahasen Amin ${ }^{1}$ and Esraa Ahmed ${ }^{2}$ \\ ${ }^{I}$ Nuclear Medicine Department, Faculty of Medicine, Cairo University, Egypt \\ ${ }^{2}$ Nuclear Medicine Department, NCI, Cairo University, Egypt \\ ${ }^{3}$ Nuclear Medicine Department, Shark El-Madina Hospital, Alex, Egypt
}

\begin{abstract}
Purpose: To investigate the impact of quantitative FDG-Metabolic activity of non-iodine avid Loco-regional recurrence [LRR; thyroid recurrence and/or cervical node metastasis] on therapy selection in thyroid cancer patients with elevated thyroglobulin [TG].

Methods: Forty thyroid cancer [33 papillary and 7 follicular] patients who underwent FDG PET/CT were studied [with $\mathrm{TSH}>30 \mathrm{mU} / \mathrm{L}$ ]. Those with only LRR were classified according to maxSUV [cut-off level 5] into high and low metabolic activity lesions [HMA \&LMA]; their therapy outcome was compared with histopathologic findings and/or follow-up routine evaluation.

Results: Only LRRs was found in 20 patients [17 papillary and 3 follicular] with diagnostic accuracy of FDG PET/CT of $100 \%$. 14/20 patients belonged to HMA where surgical neck exploration was done while in the remaining 6 patients with LMA empirical high dose of radioactive iodine-131 was given based on the assumption of the presence of a mixture of undifferentiated and differentiated thyroid cancer cells; though their post-therapy scan was negative but declined TGlevels were elicited in their follow-up [base-line $27.7 \pm 2.4$ and at follow-up $6.8 \pm 1.4 \mathrm{ng} / \mathrm{ml} ; P 0.02$ ].

Conclusion: Max-SUV based classification of non-iodine avid LRR might improve the diagnostic accuracy FDG PET/CT in a therapeutically relevant way in DTC-patients by precisely localizing them with subsequent surgical guidance in HMA lesions while those with LMA could benefit from further RA-131 therapy.
\end{abstract}

Keywords: FDG metabolic activity, FDG PET/CT, max-SUV, thyroid cancer, undifferentiated thyroid cancer.

\section{INTRODUCTION}

Differentiated thyroid cancer [DTC] occurs in 3-5 per 100,000 people and represents about $1 \%$ of all malignant tumors [1]. In most cases, prognosis is favorable and treatment consists of primary surgery followed by ablative radioiodine administration with a 10 -y survival rate of $80 \%-$ $90 \%$ [2, 3]. However, special subgroups of patients demonstrate a less favorable course of disease with much lower survival rates where poor radioiodine accumulation is usually associated [4] and presence of distant metastasis [5]. Patients with DTC may have only iodine-negative tumor lesions or both iodine-negative and iodine-positive tumor tissue $[4,6]$. Consequently, the presence of iodine-negative tumor tissue decreases the accuracy of iodine scintigraphy. This can lead to the situation in which tumor tissue is not detected by iodine scintigraphy and will remain without further treatment [7].

Such a group had less or de-differentiated thyroid cancer cells, which are more aggressive in their clinical behavior and have a high glucose metabolism and FDG uptake [8].

*Address correspondence to this author at the Nuclear Medicine Department, Faculty of Medicine, Cairo University, Post address: 32, Soliman Abaza St. Al-Mohandeseen, 11123, Giza, Egypt;

Tel: +2-010-1648583; Fax:+20233-44-45; E-mail: amramin67@gmail.com
Hence, 18F-FDG PET enables detection and precise localization of loco-regional recurrence and distant metastases in such patients with significant impact on patients' management, therefore, it was recommended as a routine diagnostic tool $[7,9]$.

18F-FDG PET for DTC has concentrated on patients with negative radioiodine scintigraphy and increased thyroglobulin (TG). Iagaru and Razfar et al. studies [10,11] demonstrated a significant difference in the mean TG in patients with positive $v s$ negative FDG PET-CT while Hamy et al. [12] recommended cervical re-exploration in patients with persistently elevated $\mathrm{TG}$ as regional recurrence is highly predicted. FDG PET-CT allows anatomical localization of non-131 I-avid surgically resectable tumors and preventing un-necessary surgical exploration with sensitivities and specificities of $85 \%$ and $94 \%$ respectively $[7,13-16]$ together with its ability to detect unrecognized distant metastatic disease.

If iodine-negative tumor sites exist in addition to iodinepositive sites, the benefit of further radioiodine therapy is questionable $[17,18]$. The importance of FDG PET-CT lies in the fact that thyroid cancer cells in patients with negative 131I result are usually more poorly or de-differentiated. As stated earlier, poorly or de-differentiated thyroid cancer cells may not take up 131I, thus making them not amenable for 
high dose 131I therapy [19]. We have an assumption that a mixture of de- and differentiated cancer cells might co-exist and the former cells will induce FDG uptake while the latter though are amenable for 131I uptake but are beyond scintigraphic resolution and can induce lower FDG uptake. Hence, we proposed in this study the term low metabolic activity [LMA] lesions to describe our suggestion and to evaluate the 131I usefulness in such situations.

\section{MATERIALS AND METHODS}

\section{Patients and Design of Study}

This prospective study was approved by the local ethics committee of the scientific review board of Cairo University and informed consent was obtained from all participants according to the Helsinki Declaration of 1964, amended in 1975 and 1983, of the World Medical Association. We included patients with DTC who were suspected of having cervical iodine-negative tumor tissue. Each patient had to meet the following inclusion criteria: elevated TG level in the euthyroid and also the hypothyroid state $(>2 \mathrm{ng} / \mathrm{mL})$ or morphologically suspected tumor disease (sonography, CT, MRI), L-thyroxin withdrawal for 4 weeks before 131-I whole-body scintigraphy, thyroid-stimulating hormone increase of $>30 \mathrm{mU} / \mathrm{L}$, performance of 131I whole-body scintigraphy meeting highquality requirements with decision of a nuclear medicine physician that no pathologic accumulation of radioiodine could be detected on whole-body scintigraphy, previous total thyroidectomy and radioiodine ablation. Exclusion criteria were: positive test for TG antibodies, administration of CT-contrast within the last 6 months, positive iodine urine test performed on the day of scintigraphy, pregnancy, or an age less than $18 \mathrm{y}$ and those with distant metastasis to bones, lungs, brain or elsewhere. FDG PET/CT was compared with histopathology [Post-surgical excision] and follow-up results as a gold standard. All data were acquired with a combined PET/CT in-line system [Biograph; Siemens Medical Solutions]. This system integrates a PET scanner (Ecat HR1; Siemens) with a dual-section helical CT scanner [Somatom; Siemens] and allows the acquisition of coregistered $\mathrm{CT}$ and PET images in one session.

\section{Imaging Modalities}

\section{Diagnostic 131-I Scan Acquisition Protocol}

131-I in doses of $185 \mathrm{MBq}$ [5mCi] were given orally to all patients and they were scanned at a mean of $48.3 \pm 1.0 \mathrm{~h}$ later. The diagnostic scanning included anterior and posterior wholebody images with spot anterior views of the neck. The scans were obtained with large field-of-view dual-head gamma cameras (Philips-Axis, Eindhoven, the-Netherlands) mounted with a high-energy parallel-hole collimator. All scans were obtained with a $20 \%$ energy window centered at $364 \mathrm{keV}$. Anterior and posterior whole-body scans were obtained with the patient supine using a $256 \times 256$ matrix and an $8.75 \mathrm{~cm} / \mathrm{min}$ scanning speed, whereas the spot view was obtained using a large field of view and a 256x256 matrix for $10 \mathrm{~min} / \mathrm{view}$.

\section{F-FDG PET/CT Acquisition Protocol}

The patients fasted at least $4 \mathrm{~h}$ before injection of 370 MBq 18F-FDG. Scanning was started 60-90 min after the injection [5-7 bed positions; acquisition time, $5 \mathrm{~min} / \mathrm{bed}$ position]. Blood glucose levels did not exceed $150 \mathrm{mg} / \mathrm{dL}$ $[8.3 \mathrm{mmol} / \mathrm{L}]$. No intravenous contrast agent was administered. Initially, patients were examined in the supine position with arms elevated, and CT scanning was started at the level of the Cervico-thoracic region with the following parameters: $40 \mathrm{mAs}$; $130 \mathrm{kV}$; slice thickness, $2.5 \mathrm{~mm}$; pitch, 1.5. The CT scans were acquired during breath holding within the normal expiration position and reached caudally to the mid-thigh. PET over the same region was performed immediately after acquisition of the CT images $[5 \mathrm{~min} / \mathrm{bed}$ position]. Afterward, a second data acquisition set from the base of the skull to the upper thoracic region was initiated with the patient's arms positioned next to the body. For this second part of the examination, the same parameters were used for CT and PET. The CT data were used for attenuation correction, and images were reconstructed as $5-\mathrm{mm}$ slices applying a standard iterative algorithm.

\section{FDG PET CT Interpretation}

Images were interpreted at a workstation equipped with fusion software [Syngo; Siemens] that provides multi-planar reformatted images and enables display of the PET images [with and without attenuation correction], CT images, and fused PET/CT images in any percentage relation. Analysis of images was performed as a multistep image interpretation [20]. During the first step of analysis, PET/CT and CT images were scored blindly and independently by 2 nuclear medicine physicians and 2 radiologists. For this purpose, we adopted the diagnostic scale of Palmedo et al. [4].

A lesion was considered as true-positive if the score was 2-4 and if histopathology was positive or if it showed progression at follow-up sessions. Findings were considered as true-negative if histology was negative or if follow-up examinations did not show any progression in the region of concern for at least 12 months. A lesion was considered as false-positive if histopathology was negative or if it showed no progression at follow-up sessions. Those considered as false-negative if histology was positive or if follow-up examinations showed growth of the lesion(s) [4]. Follow-up examinations were performed every 3-6 months and included measurement of TG levels. The terms of locoregional recurrence [either in the thyroid bed or cervical adenopathy or both] were used. In our study, in the analysis of FDG PET/CT the term LMA was used to describe those with $\operatorname{maxSUV} \leq 5$ where we assume a mixture of de- and differentiated cells in cervical lesions could be present, while those $>5$ termed High metabolic activity [HMA].

\section{Therapeutic Procedure}

After FDG PET/ imaging-based localization of active lesions; surgical excision was carried out in HMA cases. Those with LMA lesions were shifted to empirical high dose of I131 therapy [150 mCi].

\section{Statistical Analysis}

Data were statistically described in terms of mean \pm standard deviation $( \pm \mathrm{SD})$, median and range, or frequencies (number of cases) and percentages when appropriate. ANOVA was used for statistical comparative analysis. The 
diagnostic accuracy of PET/CT was calculated on the basis of the true-positive and true-negative findings as described. $\boldsymbol{P}$ value less than 0.05 was considered significant althrough.

\section{RESULTS}

\section{Patients' Population}

Forty patients were included in the study. The age of our patients ranged 22-76 years with median and mean ages of 48 years and $47.5 \pm 16.2$ respectively. There is a predominance of disease in females with female to male ratio approximately $3: 1$. There was a high frequency of papillary carcinoma found in 33 patients [82.5\%] while 7 [17.5\%] had follicular carcinoma. TG at study entry ranged $20-66 \mathrm{ng} / \mathrm{ml}$ with a mean of $37.7 \pm 15.4$. All of these patients underwent previous total thyroidectomy and ablative radioiodine treatment. Histology and follow-up [18 months $\pm 6.2]$ served as the gold standard. The cancer was staged according to the American Joint Committee on Cancer [18].

\section{FDG PET CT Analysis}

20 out of the studied 40 patients were excluded; 4 because of a negative FDG PET CT and 16 due to the finding of distant deposits. The remaining 20 patients [17 papillary and 3 follicular; mean age $43.5 \pm 13.2]$ were positive for loco-regional recurrence. 14/20; 70\% [5/14 patients with thyroid recurrence while metastatic adenopathy in all of them] belonged to HMA group [mean maxSUV 8.1 \pm 2.3 ] while the remaining 6 patients [30\%] showed LMA in cervical adenopathy. In the former group all of them were operated and proved to be positive on histo-pathology with evidence of decline of their follow-up TG [mean before surgery $39.7 \pm 10.4$ and at follow-up $7.7 \pm 1.4 ; \boldsymbol{P} 0.03$ ], while in LMA group high empirical I131 dose was given [150 $\mathrm{mCi}$ ] and reduced follow-up TG was elicited [base-line $27.7 \pm 2.4$ and at follow-up $6.8 \pm 1.4 ; \boldsymbol{P} 0.02]$, though their post-therapy scans were negative. The 6/20 LMA patients were 5 females and one male; mean ages $35 \pm 4.1$ years, papillary type, with their initial diagnosis of T1-2NOM0. Regarding the levels of cervical LNs deposits; level IV was the commonest site [30\%] followed by involvement of level II, III and VI [ $15 \%, 15 \%$ and $28 \%$ respectively] and the least at level V [12\%]. Diagnostic accuracy of FDG PET/CT was $100 \%$ based on either histopathology or follow-up.

\section{DISCUSSION}

This study was carried out on 40 DTC patients with negative iodine-131 diagnostic scan and elevated TG. FDG PET/CT was done in all the studied cases and showed a high prevalence of only LRR $[20 / 40 ; 50 \%$ ] with subsequent impact on the selection of management either surgical or radioactive iodine-131. Besides, as far as we know no previous studies have focused on RAI-131 therapy in patients with what we termed as LMA [6/20 cases] where empirical high dose of RAI-131 showed a beneficial outcome as proven by significant decline of their follow-up TG.

Patients with DTC have a less favorable prognosis if radioiodine-negative tumor tissue, especially in distant metastases, is present. Also, these cancers are not detected by routine diagnostic work-up with radioactive iodine-131 scintigraphy and they cannot be treated efficiently by radioiodine therapy. Therefore, tumor cells continue to grow undetected and the chance of cure decreases significantly [4]; hence the most effective therapy is surgical excision of these lesions.

18F-FDG PET is able to reveal iodine-negative tumor tissue with a diagnostic accuracy that is higher than that of other imaging modalities [7]. In this study, the diagnostic accuracy was $100 \%$ in detection of LRR. These values are within the range of the diagnostic accuracies that have been reported by other studies $[6,7,14-16,21,22]$. In fact FDG $\mathrm{PET} / \mathrm{CT}$ leads to exact localization of hypermetabolic lesions resulting in higher diagnostic confidence, compared with each imaging modality alone "CT and PET" [23-26]. Furthermore, pitfalls of PET are reduced using PET/CT [27].

The main limitation of $18 \mathrm{~F}-\mathrm{FDG}$ PET is the possibility of false-positive 18F-FDG accumulations in the neck leading to false diagnosis of lymph node metastases and the potential scheduling of a futile operation [28, 29]. However, this was not the case in our study regarding the cervical region as experience and knowledge of normal physiological FDG uptake are established and well accounted by the interpretators as in FDG uptake in vocal cords that was misinterpreted in some of the studied cases in Palmedo et al. study [4].

The main aspect of the study is evaluation of its therapeutic relevance of FDG PET/CT on the investigated patients. The optimal for patients with iodine-accumulating tumors is surgical removal followed by radioactive-iodine ablation [30]; however this is appropriate if only iodinenegative tumors are present where complete surgical removal of iodine-negative tissue is the only curative treatment option [4]. Furthermore, it seems important to diagnose and remove iodine-negative tumor tissue as early as possible because prognosis of this tumor subgroup is essentially worse than that of the other scenarios of differentiated thyroid cancer [13, 31-33].

FDG-PET can be used in patients with known iodinepositive tumor sites to localize coexisting additional iodinenegative tumor tissue that is FDG-positive [17]. If iodinenegative tumor sites exist in addition to iodine-positive sites, the benefit of further radioiodine therapy is questionable [17, 18]. In our study 6 patients received empirical high dose of radioactive iodine-131 with negative post-therapy scan. Though, it was reported that high-dose radioiodine therapy appears to have little or no effect on iodine-negative FDGavid metastatic lesions [34], but in those patients we noticed maxSUV was $\leq 5$ in FGD PET/CT and we consider them as of LMA that could be attributed to the presence of a less differentiated or mixed components of well and undifferentiated cancer tissues in the positive lesions, hence we decide to give empirical high-dose of radioactive iodine-131 and evaluate its potential usefulness. The latter was confirmed by the declined TG found in their follow-up however such lesions were beyond the scintigraphic resolution of post-therapy iodine-131 scan. In fact though post-therapy TG level was $6.8 \pm 1.4 \mathrm{ng} / \mathrm{ml}$ but this was acceptable as it was stated that: 
"for patients with a follow-up TG level between 2.0 and $8.0 \mathrm{ng} / \mathrm{mL}$, we can consider these patients truenegative clinical findings if the patients are stable over a minimum follow-up of 1 year" [11].

However, for further confirmation of our suggestion, further study with larger sample size should be performed on such a patient sub-group.

Other points of some concern are the limitations of our study; First: CT is usually performed without a contrast agent in such group. However, in patients with DTC, CT is generally done without the application of a contrast agent to avoid blockage of iodine uptake. This also applies to patients in whom iodine-negative tissue is suspected, because iodinenegative and iodine-positive tissue may coexist in one patient [4]. Second, the minimal time for follow-up of patients [ $18 \pm 6.2$ months] through $18 \mathrm{~F}-\mathrm{FDG}$ avidity is a predictor of reduced survival $[4,35]$; hence our study did not determine the long term outcome of applied strategy of therapy.

Finally, we consider our suggestion regarding the cervical adenopathy with what we call LMA in DTC patients with negative iodine-131 scan and elevated TG could give the opportunity for I131 therapy in such patients from the start and avoidance of serious surgical morbidity if these lesions are located in the vicinity of critical structures as great vessels.

\section{CONCLUSION}

Integrated FDG PET/CT has an excellent diagnostic accuracy in a therapeutically relevant way in thyroid cancer patients with iodine-negative loco-regional recurrences by precisely localizing tumor tissue and guiding the clinical management of recurrent thyroid carcinoma and aids in the selection of appropriate salvage. What could be added from the current study is that those with LMA could benefit from further empirical high dose of RA-131 therapy as a less differentiated or a mixture of un-differentiated thyroid cancer cells could be present but further studies with a larger patients' number are needed to support this suggestion.

\section{CONFLICT OF INTEREST}

The authors state no conflict of interest.

\section{ACKNOWLEDGEMENTS}

The authors thank Dr. Zeinab Nawito for helpful contributions to the grammatical aspects of this work.

\section{REFERENCES}

[1] Stewart BW, Kleihues P. Thyroid cancer. In: Stewart BW, Kleihues P, eds. World Cancer Report. Lyon, France: Lyon IARC Press; 2003: pp. 257-60.

[2] AACE Clinical Practice Guidelines for the Management of Thyroid Carcinoma. (http://www.aace.com/clin/guidelines/thyroid_carcino ma.pdf).

[3] Gilliland FD, Hunt WC, Morris DM, et al. Prognostic factors for thyroid carcinoma: a population-based study of 15,698 cases from the Surveillance, Epidemiology and End Results (SEER) program 1973-1991. Cancer 1997; 79: 564-73.
[4] Palmedo H, Bucerius J, Joe A, et al. Integrated PET/CT in differentiated thyroid cancer: diagnostic accuracy and impact on patients' management. J Nucl Med 2006; 47: 616-24.

[5] Sisson JC, Giordano TJ, Jamadar DA, et al. 131-I treatment of micronodular pulmonary metastases from papillary thyroid carcinoma. Cancer 1996; 78: 2184-92.

[6] Grunwald F, Schomburg A, Bender H, et al. Fluorine-18 fluorodeoxyglucose positron emission tomography in the follow-up of differentiated thyroid cancer. Eur J Nucl Med 1996; 23: 312-9.

[7] Grunwald F, Menzel C, Bender H, et al. Comparison of F-18 FDGPET with iodine-131 and Tc-99m-sestamibi scintigraphy in differentiated thyroid cancer. Thyroid 1997; 7: 327-35.

[8] Khan N, Oriuchi N, Higuchi T, Zhang H, Endo K. PET in the follow-up of differentiated thyroid cancer. Br J Radiol 2003; 76: 690-5.

[9] Bannas P, Derlin T, Groth M, et al. Can (18) F-FDG-PET/CT be generally recommended in patients with differentiated thyroid carcinoma and elevated thyroglobulin levels but negative I-131 whole body scan? Ann Nucl Med 2012; 26: 77-85.

[10] Iagaru A, Kalinyak JE, McDougall IR. F-18 FDG PET/CT in the management of thyroid cancer. Clin Nucl Med 2007; 32(9): 690-5.

[11] Razfar A, Branstetter BF 4th, Christopoulos A, et al. Clinical usefulness of positron emission tomography computed tomography in recurrent thyroid carcinoma. Arch Otolaryngol Head Neck Surg 2010; 136(2): 120-5

[12] Hamy A, Mirallie' E, Bennouna J, et al. Thyroglobulin monitoring after treatment of well-differentiated thyroid cancer. Eur J Surg Oncol 2004; 30(6): 681-5.

[13] Wang W, Macapinlac H, Larson SM, et al. [18F]-2-fluoro-2-deoxyD-glucose positron emission tomography localizes residual thyroid cancer in patients with negative diagnostic 131I whole body scans and elevated serum thyroglobulin levels. J Clin Endocrinol Metab 1999; 84: 2291-302.

[14] Scott GC, Meier DA, Dickinson CZ. Cervical lymph node metastasis of thyroid papillary carcinoma imaged with fluorine-18FDG, technetium-99m-pertechnetate and iodine-131-sodium iodide. J Nucl Med 1995; 36: 1843-5.

[15] Chung JK, So Y, Lee JS, et al. Value of FDG PET in papillary thyroid carcinoma with negative I-131 whole-body scan. J Nucl Med 1999; 40: 986-92.

[16] Feine U, Lietzenmayer R, Hanke JP, et al. Fluorine-18-FDG and iodine-131-iodide uptake in thyroid cancer. J Nucl Med 1996; 37 1468-72.

[17] Grebe SK, Hay ID. Thyroid cancer nodal metastases: biologic Significance and therapeutic considerations. Surg Oncol Clin N Am 1996; 5: 43-63

[18] Vassilopoulou-Sellin R, Schultz PN, Haynie TP. Clinical outcome of patients with papillary thyroid carcinoma who have recurrence after initial radioactive iodine therapy. Cancer 1996; 78: 493-501.

[19] Lee PI, You DL, Hsu YL, Chen J, Huang YY, Wu YY. FDG PET$\mathrm{CT}$ in patients with recurrent thyroid cancer with elevated serum thyroglobulin but negative iodine-131 whole body scan. Ann Nucl Med Sci 2007; 20: 179-18

[20] Lardinois D, Weder W, Hany TF, et al. staging of non-small-cell lung cancer with integrated positron-emission tomography and computed tomography. N Engl J Med 2003; 348: 2500-7.

[21] Cooper DS, Doherty GM, Haugen BR, et al. American thyroid association (ATA) guidelines taskforce on thyroid nodules and differentiated thyroid cancer. Revised American thyroid association management guidelines for patients with thyroid nodules and differentiated thyroid cancer. Thyroid 2009; 19: 1167-214.

[22] Lee PI, You DL, Hsu YL, Chen J, Huang YY, Wu YY. FDG PET$\mathrm{CT}$ in patients with recurrent thyroid cancer with elevated serum thyroglobulin but negative iodine-131 whole body scan. Ann Nucl Med Sci 2007; 20: 179-18.

[23] Nahas Z, Goldenberg D, Fakhry C, et al. The role of positron emission tomography/computed tomography in the management of recurrent papillary thyroid carcinoma. Laryngoscope 2005; 115(2): 237-43.

[24] Wang W, Macapinlac H, Larson SM, et al. [18F]-2- fluoro-2deoxy-D-glucose positron emission tomography localizes residual thyroid cancer in patients with negative diagnostic (131)I whole body scans and elevated serum thyroglobulin levels. J Clin Endocrinol Metab 1999; 84(7): 2291-302. 
[25] Chung JK, So Y, Lee JS, et al. Value of FDG-PET in papillary thyroid carcinoma with negative 131I whole-body scan. J Nucl Med 1999; 40(6): 986-92.

[26] Schluter B, Bohuslavizki KH, Beyer W, et al. Impact of FDG-PET on patients with differentiated thyroid cancer who present with elevated thyroglobulin and negative 131I scan. J Nucl Med 2001; 42(1): 71-6.

[27] Burger I, Gorres G, von Shulthess G, et al. PET/CT: diagnostic improvement in recurrent colorectal carcinoma compared to PET alone. Radiology 2002; 225:S424.

[28] Schoder H, Erdi YE, Larson SM, et al. PET/CT: a new imaging technology in nuclear medicine. Eur J Nucl Med Mol Imaging 2003; 30(10): 1419-37.

[29] Lind P, Kohlfurst S. Respective roles of thyroglobulin, radioiodine imaging, and positron emission tomography in the assessment of thyroid cancer. Semin Nucl Med 2006; 36(3): 194-205.
[30] Sisson JC, Ackermann RJ, Meyer MA, et al. Uptake of 18-fluoro2-deoxy-D-glucose by thyroid cancer: implications for diagnosis and therapy. J Clin Endocrinol Metab 1993; 77: 1090-4.

[31] Dietlein M, Scheidhauer K, Voth E, et al. Fluorine-18 fluorodeoxyglucose positron emission tomography and iodine-131 whole-body scintigraphy in the follow-up of differentiated thyroid cancer. Eur J Nucl Med 1997; 24: 1342-8.

[32] Hay ID, Grant CS, Bergstralh EJ, et al. Unilateral total lobectomy: is it sufficient surgical treatment for patients with AMES low-risk papillary thyroid carcinoma? Surgery 1998; 124: 958-64.

[33] Grebe SK, Hay ID. Thyroid cancer nodal metastases: biologic Significance and therapeutic considerations. Surg Oncol Clin N Am 1996; 5: 43-63.

[34] Vassilopoulou-Sellin R, Schultz PN, Haynie TP. Clinical outcome of patients with papillary thyroid carcinoma who have recurrence after initial radioactive iodine therapy. Cancer 1996; 78: 493-501.

[35] Sundram F. Clinical use of PET/CT in thyroid cancer diagnosis and management. Biomed Imaging Interv J 2006; 2(4): e56.

Received: March 7, 2014

Revised: June 7, 2014

Accepted: June 25, 2014

(C) Amin et al.; Licensee Bentham Open.

This is an open access article licensed under the terms of the Creative Commons Attribution Non-Commercial License (http://creativecommons.org/licenses/by-nc/3.0/) which permits unrestricted, non-commercial use, distribution and reproduction in any medium, provided the work is properly cited. 